\title{
Collective modes in flux line liquids
}

To cite this article: B Tanatar and A Oral 2000 Supercond. Sci. Technol. 13968

\section{Related content}

- Effects of disorder on collective modes in
Bingle- and double-layer Bose systems
B Tanatar and A K Das
- Collective excitations and screened
$\frac{\text { interactions in two-dimensional charged }}{\text { Bose systems }}$
B Tanatar and A K Das
- Monte Carlo simulation of the crossover
$\frac{\text { from Bose glass to Bragg glass phase in }}{\text { layered BSCCO with columnar defects }}$
L M Queiroz and M D Coutinho-Filho

View the article online for updates and enhancements. L M Queiroz and M D Coutinho-Filho

\section{IOP ebooks}

Bringing you innovative digital publishing with leading voices to create your essential collection of books in STEM research. Start exploring the collection - download the first chapter of every title for free. 


\title{
Collective modes in flux line liquids
}

\author{
B Tanatar and A Oral \\ Department of Physics, Bilkent University, Bilkent, 06533 Ankara, Turkey
}

Received 6 November 1999, in final form 21 February 2000

\begin{abstract}
We study the collective modes of flux line liquids such as occur in the type-II superconductors of recent interest composed of two-dimensional layered structures. Starting from the vortex-vortex interaction and employing the dielectric formalism within the random-phase approximation, we find propagating sound modes in the long-wavelength limit.
\end{abstract}

The discovery of high-temperature superconductivity has led to a renewed interest in the properties of type-II superconductors. Magnetic flux lines penetrating the superconductor form the so-called Abrikosov lattice and various properties of these structures are discussed in detail in recent reviews [1,2]. The materials of recent interest are highly anisotropic with conducting two-dimensional copper oxide planes, separated by effectively insulating layers. Thus, a magnetic field perpendicular to the planes produces quantized flux lines which may be considered as stacks of pancake vortices confined to the conducting planes. The static and dynamic properties of these vortices have been the subject of intense research activity. Fluctuations in the vortex system give rise to a transition from the solid-like structure to a liquid-like phase, as has been observed experimentally [3,4]. Moreover, it is still controversial whether the flux lines decouple into a liquid of flux pancakes above the melting line or not. Recent experiments continue to explore the melting transition in flux line lattices in different materials, effects of disorder, and application possibilities [5]. For instance, it has been observed that the disorder does not affect the melting of the flux line lattices and leads to the coexistence of solid and liquid phases on a microscopic scale [6].

The melting of the flux line lattice and its properties have been considered theoretically [1]. For the description of the fluid phase either the boson analogy of Nelson [7] is used [8,9], or the classical liquid-state theory is employed [10-12]. Since a number of works make use of the ground-state correlation functions of the flux line liquid to determine the solid-fluid phase boundary, our aim in this work is to study the collective modes in the flux line liquids. Theoretical approaches calculating the structure factors in the liquid include the hypernetted-chain approximation, molecular dynamics, and Monte Carlo simulations. We use the dielectric formulation to describe the long-wavelength collective excitations in the flux line liquid. Modelling the system as a liquid interacting with a pairwise interaction potential at a given temperature and density in the absence of any disorder, we calculate the density-density response of the liquid within the random-phase approximation.

The collective modes of flux line lattices have attracted considerable attention, especially ones for which Josephson coupling is present $[4,13]$. In the case of zero or negligible
Josephson tunnelling between the superconducting planes, the collective excitations are also determined. Fetter [14] has calculated the normal modes and specific heat of pancake vortices in superconducting multilayers. In contrast, the behaviour of the collective modes of melted flux lattices, namely the flux line liquids, has received less attention. The motion of vortices in a superconductor is generally overdamped and there are no sound waves in the system. Marchetti and Nelson [15] used a hydrodynamic theory to study the collective modes in isotropic and hexatic flux line liquids. They find propagating modes that are proportional to $q^{2}$. In our approach, we assume a liquid state (in the clean limit) for the flux lines and arrive at sound modes.

In the following, we introduce the interaction potential for flux lines in the liquid state, and employ the dielectric formalism to calculate the collective modes of the system in the lowest-order approximation scheme. We assume infinite anisotropy, i.e. $M_{c} \rightarrow \infty$, in which case the vortex-vortex interaction can be expressed as [16]

$$
\beta V\left(q, q_{z}\right)=\frac{\Gamma \lambda^{2}\left[q^{2}+\left(4 / d^{2}\right) \sin ^{2}\left(q_{z} d / 2\right)\right]}{q^{2}\left[1+\lambda^{2} q^{2}+4\left(\lambda^{2} / d^{2}\right) \sin ^{2}\left(q_{z} d / 2\right)\right]}
$$

where $\beta=1 / k_{B} T$, and $\Gamma=\beta d \Phi_{0}^{2} /\left(4 \pi \lambda^{2}\right)$ is the dimensionless coupling constant. Here, $\Phi_{0}$ is the flux quantum, $d$ is the interplane distance, and $\lambda$ is the penetration depth. $\quad q$ and $q_{z}$ denote the in-plane and out-of-plane wave vectors, respectively. This model is equivalent to assuming that the superconducting layers are decoupled, i.e. interlayer Josephson coupling vanishes. In practice, this condition holds for BSCCO or artificially produced multilayer materials [17], and the above law of interaction between the flux lines is employed in a variety of liquid-state calculations to determine the properties of melted flux line liquids.

Noting the similarity between the present situation of pancake vortices and layered semiconductors [18], we exploit the same dielectric formalism. The density-density response function for the anisotropic flux liquid within the randomphase approximation (RPA) is given by

$$
\chi\left(q, q_{z}, \omega\right)=\frac{\chi_{0}^{2 \mathrm{D}}(q, \omega)}{1-V\left(q, q_{z}\right) \chi_{0}^{2 \mathrm{D}}(q, \omega)}
$$


where $\chi_{0}^{2 \mathrm{D}}(q, \omega)$ is the noninteracting response function in the plane. The situation here is very similar to that of the layered semiconducting structures, for which a whole body of theoretical approaches for determining the collective plasma oscillations exist $[18,19]$. The collective modes of the layered system are obtained by solving the equation

$$
1-V\left(q, q_{z}\right) \chi_{0}^{2 \mathrm{D}}(q, \omega)=0 .
$$

The density-density response function of a classical 2D system is given by $[20,21]$ (we take $\hbar=1$ )

$$
\chi_{0}^{2 \mathrm{D}}(q, \omega)=-n \beta W\left(\frac{\omega}{q}\left(\frac{M_{\|}}{k_{B} T}\right)^{1 / 2}\right)
$$

where

$$
W(z)=\frac{1}{\sqrt{2 \pi}} \int_{-\infty}^{\infty} \mathrm{d} x \frac{x \mathrm{e}^{-x^{2} / 2}}{x-z-\mathrm{i} \eta}
$$

is the plasma dispersion function [21] ( $\eta$ is a positive, infinitesimal quantity). In the calculation of the response function, single-particle energy $p^{2} / 2 M_{\|}$is assumed for the vortices, where $M_{\|}$is the in-plane effective mass.

Using the asymptotic properties of the plasma dispersion function, in the long-wavelength limit and for small damping we obtain the dispersion of the collective modes as

$$
\omega^{2}\left(q, q_{z}\right)=\frac{n}{M_{\|}} q^{2} V\left(q, q_{z}\right)\left[1+\frac{3 k_{B} T}{n V\left(q, q_{z}\right)}\right] .
$$

In particular, we find propagating sound waves along the $x y$ and $z$-directions, with dispersion relations

$$
\omega\left(q, q_{z}=0\right) \simeq\left(n \Gamma / M_{\|} \beta\right)^{1 / 2} \lambda q
$$

and

$$
\omega\left(q=0, q_{z}\right) \simeq\left(n \Gamma / M_{\|} \beta\right)^{1 / 2} \lambda q_{z}
$$

respectively. The London penetration depth is assumed to have a temperature dependence of the form

$$
\lambda(T)=\lambda_{0} /\left[1-\left(T / T_{c}\right)^{4}\right]^{1 / 2}
$$

with $\lambda_{0}=1500 \AA$ and $T_{c}=85 \mathrm{~K}$ for BSCCO materials. These modes are largely independent of the temperature (in the long-wavelength limit, and away from the melting line), since $\Gamma \sim \beta$. If we choose $q_{z} d=\pi$, the collective mode dispersion in the long-wavelength limit $(q \rightarrow 0)$ becomes

$\omega\left(q \rightarrow 0, q_{z}=\pi / d\right) \simeq\left(n \Gamma / M_{\|} \beta\right)^{1 / 2} 2 \lambda / 2\left(1+4 \lambda^{2} / d^{2}\right)^{1 / 2}$

displaying an optical mode. Thus, the anisotropic nature of the layered superconducting systems give rise to a variety of collective mode dispersions for the melted flux line liquids, similar to the situation for layered semiconducting materials.

We plot the collective mode dispersions in a flux line liquid in figure 1 at $B=1 \mathrm{~T}$ and $T=60 \mathrm{~K}$. We scale the wave vectors $q$ and $q_{z}$ by the average interparticle distance in the plane $a$, defined by the relation $B / \Phi_{0}=$ $1 /\left(\pi a^{2}\right)$. We also need the effective mass $M_{\|}$, which is not known precisely for BSCCO materials from the available experiments. Furthermore, the mass of a vortex is an issue of controversy, and there exist different approaches to its
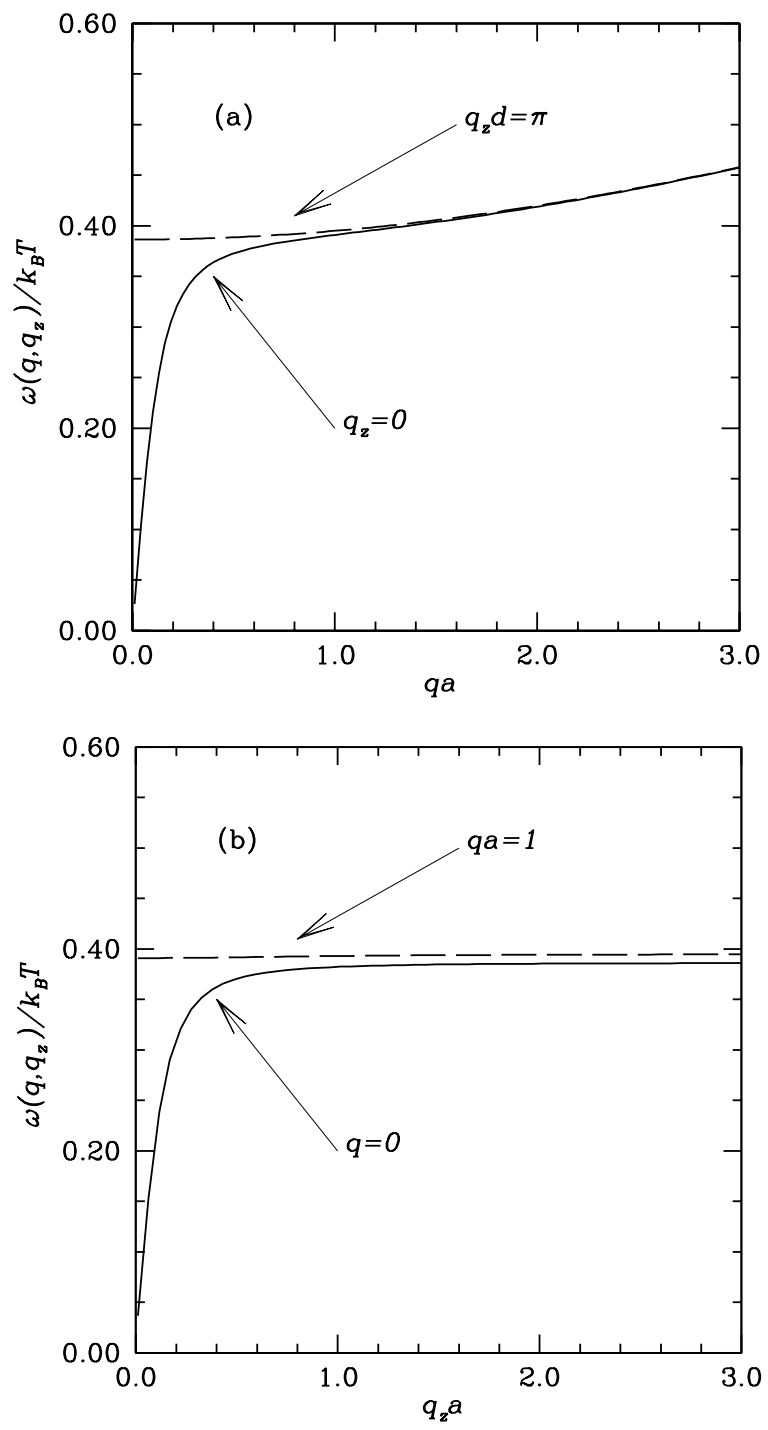

Figure 1. (a) The collective modes of a flux line liquid at $B=1 \mathrm{~T}$ and $T=60 \mathrm{~K}$ as a function of the in-plane wave vector $q$. The solid and dashed lines indicate $q_{z}=0$ and $q_{z} d=\pi$ sectors, respectively. (b) The collective modes as a function of $q_{z}$. The solid and dashed lines indicate $q=0$ and $q a=1$, respectively.

definition [1,22]. We adopt here the viewpoint that $M_{\|}$is the Ginzburg-Landau effective mass, and take $M_{\|}=10 m_{e}$, where $m_{e}$ is the bare electron mass, for illustration purposes. $\omega\left(q, q_{z}\right)$ as functions of $a$ and $q_{z}$ are depicted in figures 1(a) and $1(\mathrm{~b})$, respectively. We observe that $\omega\left(q, q_{z}=0\right)$ displays a sound-like acoustic mode in the long-wavelength limit, whereas $\omega\left(q, q_{z}=\pi / d\right)$ has an optical character. When we fix the in-plane wave vector and look at the collective modes as a function of $q_{z}$, we again find acoustic or optical mode behaviour, depending on $q$. We can make contact with the results of Norborg and Blatter [8] who used the boson analogy to simulate flux line liquids and obtained the excitation spectrum for $2 \mathrm{D}$ bosons. In the approach using the boson analogy the mapping of the vortex system to bosons turns the potential energy of the vortex system into an action for the Bose system [7]. This action completely describes the dynamics of the Bose system and the collective excitation spectrum can be determined. In the present case, on the other 
hand, a dynamic term $p^{2} / 2 M_{\|}$is added to the vortex potential energy to calculate the linear response and thus the spectrum of excitations.

Our results may be quantitatively modified by considering the effects of correlation between the flux lines within, say, the self-consistent-field approach. However, we believe that the correlation effects will only alter the large- $q$ portion of the dispersion relation, leaving the longwavelength behaviour discussed here mostly unchanged. It would be interesting to include the effects of disorder on the collective mode behaviour of flux line liquids, and investigate the structure of the modes near the melting phase boundary. We also remark that neutron scattering experiments [23] measuring the dynamic structure factor $S\left(q, q_{z}, \omega\right)$ can, in principle, be used to deduce the collective mode behaviour of flux line liquids discussed here.

\section{Acknowledgments}

This work was partially supported by the Scientific and Technical Research Council of Turkey (TUBITAK) under Grant No TBAG-1662 and NATO under Grant No SfP971970. We thank Professor I O Kulik and Dr Y-J Kim for useful discussions.

\section{References}

[1] Blatter G, Feigel'man M V, Geshkenbein V B, Larkin A I and Vinokur V M 1994 Rev. Mod. Phys. 661125

[2] Brandt E H 1995 Rep. Prog. Phys. 581465

[3] Gammel P L, Schneemeyer L F, Waszczak J V and Bishop D J 1988 Phys. Rev. Lett. 611125

[4] Muller K A, Takashige M and Bednorz J G 1987 Phys. Rev. Lett. 581143

Yeshurun Y and Malozemoff A P 1988 Phys. Rev. Lett. 60 2202
[5] Zeldov E, Majer D, Konczykowski M, Geshkenbein V B, Vinokur V M and Shtrikman H 1995 Nature 375373

Oral A, Barnard J C, Bending S J, Kaya I I, Ooi S, Taoka H, Tamegai T and Henini M 1998 Phys. Rev. Lett. 803610

[6] Oral A, Barnard J C, Bending S J, Ooi S, Tamegai T and Henini M 1997 Phys. Rev. B 5614295

[7] Nelson D R 1988 Phys. Rev. Lett. 601973

Nelson D R and Seung H S 1989 Phys. Rev. B 399153

[8] Norborg H and Blatter G 1998 Phys. Rev. B 5814556

[9] Bulutay C, Tanatar B and Tomak M 1998 Phys. Rev. B 57 15197

Stepparola E, Nifosi R and Tosi M P 1998 J. Phys.: Condens. Matter 1011645

[10] Sengupta S, Dasgupta C, Krishnamurty H R, Menon G I and Ramakrishnan T V 1991 Phys. Rev. Lett. 673444

Menon G I, Dasgupta C, Krishnamurty H R, Ramakrishnan T V and Sengupta S 1996 Phys. Rev. B 54 16192

[11] Krämer A and Díaz-Herrera J E 1998 Phys. Rev. B 588755

[12] Cornaglia P S and Balseiro C A 2000 Phys. Rev. B 61784

[13] Bulaevskii L N and Maley M P 1993 Phys. Rev. Lett. 713541 Bulaevskii L N 1996 Turk. J. Phys. 20594

[14] Fetter A L 1994 Phys. Rev. B 5013695

[15] Marchetti M C and Nelson D R 1991 Physica C 17440

[16] Feigel'man M V, Geshkenbein V B and Larkin A I 1990 Physica C 167177

[17] White W R, Kapitulnik A and Beasley M R 1991 Phys. Rev. Lett. 662826

[18] da Costa W B, de Freitas U and Studart N 1998 Phys. Status Solidi b 206661

[19] Ando T, Fowler A B and Stern F 1982 Rev. Mod. Phys. 54 437

[20] Studart N and Hipolito O 1979 Phys. Rev. A 191790

[21] Ichimaru S 1972 Basic Principles of Plasma Physics (Reading, MA: Benjamin-Cummings)

[22] Sonin E B, Geshkenbein V B, van Otterlo A and Blatter G 1988 Phys. Rev. B 57575

Kopnin N B and Vinokur V M 1998 Phys. Rev. Lett. 813952

[23] Johnson S T, Forgan E M, Lloyd S H, Aegerter C M, Lee S L, Cubitt R, Kealey P G, Ager C, Tajima S, Rykov A and Paul D McK 1999 Phys. Rev. Lett. 822792 